\title{
3D co-cultured endothelial cells and monocytes promoted cancer stem cells stemness and malignancy
}

Shupei Qiao ${ }^{\mathrm{a}, \mathrm{b}, 1}$, Yufang Zhao ${ }^{\mathrm{a}, 1}$, Hui Tian ${ }^{\mathrm{a}, 1}$, Ishara Manike ${ }^{\mathrm{a}}$, Liang $\mathrm{Ma}^{\mathrm{a}}$, Hongji Yan ${ }^{\mathrm{c}, \mathrm{d} *}$ and Weiming Tian ${ }^{\mathrm{a}, *}$

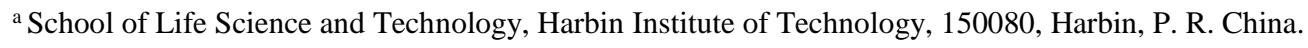

${ }^{\mathrm{b}}$ Harbin Medical University, 150080, Harbin, P. R. China.

${ }^{c}$ Division of Glycoscience, Department of Chemistry, School of Engineering Sciences in Chemistry,

Biotechnology and Health, KTH, Royal Institute of Technology, AlbaNova University Center, 10691 Stockholm, Sweden

d AIMES - Center for the Advancement of Integrated Medical and Engineering Sciences, Karolinska Institute, Department of Neuroscience/Biomedicum, 171 77, Solnavägen 9, Solna, Sweden

${ }^{1}$ Equal contribution

*Corresponding authors

E-mail: hongji@kth.se (Dr. Hongji Yan)

E-mail: tianweiming@hit.edu.cn (Prof. Weiming Tian) 


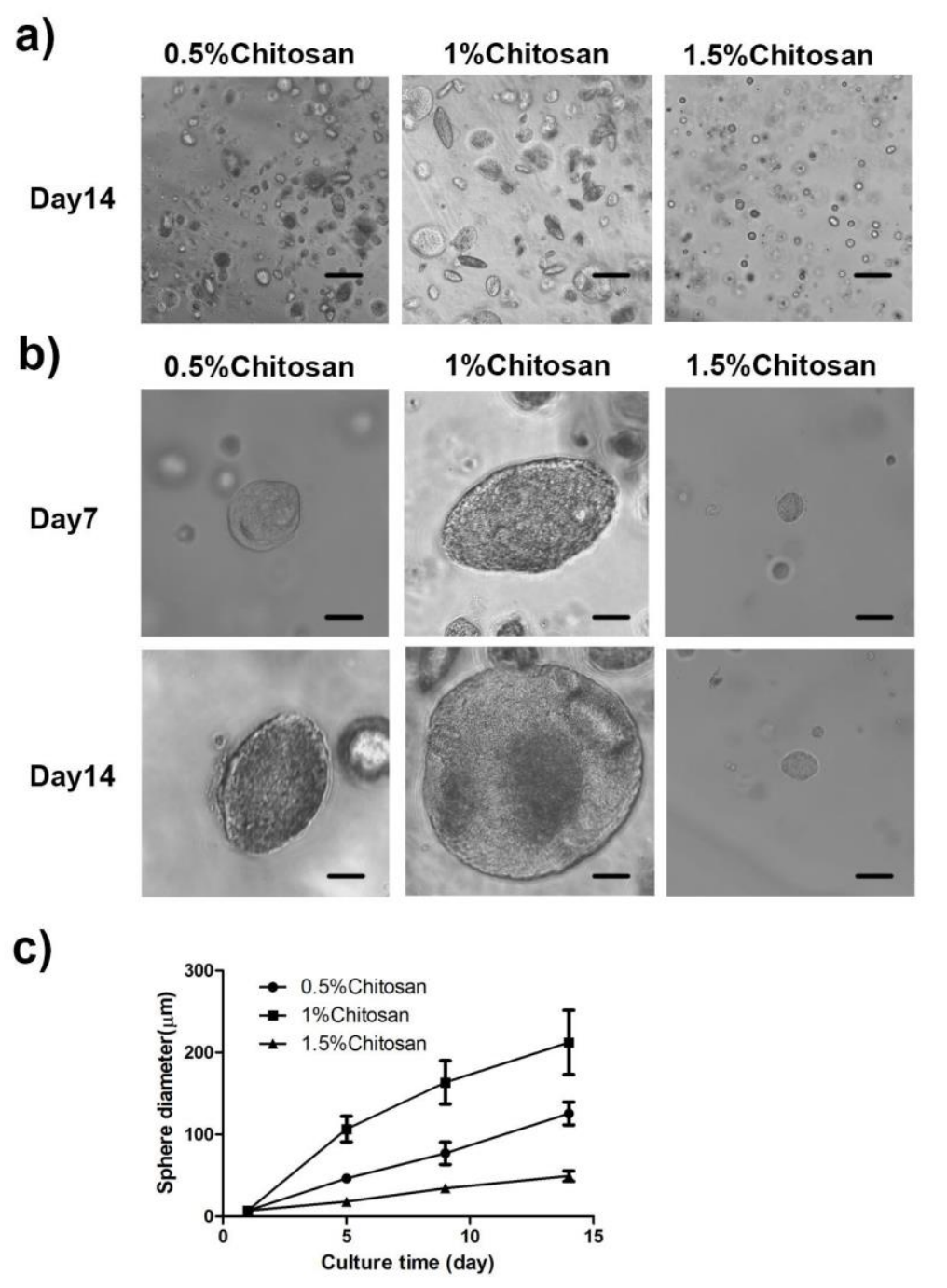

S1. Effects of hydrogels with different concentrations of chitosan on stromal cells growth and proliferation

a), b) Observation of stromal cell morphology, the scales are $500 \mu \mathrm{m}$ and $50 \mu \mathrm{m}$, respectively; c) Clone diameter statistics, the data represent the mean \pm s.e.m., $\mathrm{n}=12$; 
Supplementary Table 1 primer information for Real-time RT-PCR

\begin{tabular}{|c|c|}
\hline Gene name & Sense/antisense primers $\left(5^{\prime}-3^{\prime}\right)$ \\
\hline \multirow{2}{*}{$C D 44$} & TCGATTTGAATGTAACCTGCCG \\
\hline & CAGTCCGGGAGATACTGTAGC \\
\hline \multirow{2}{*}{$C D 24$} & GTTGCACCGTTTCCCGGTAA \\
\hline & CCCCTCTGGTGGTAGCGTTA \\
\hline \multirow{2}{*}{ MMP9 } & CTGGACAGCCAGACACTAAAG \\
\hline & CTCGCGGCAAGTCTTCAGAG \\
\hline \multirow{2}{*}{ Sox 2} & GCGGAGTGGAAACTTTTGTCC \\
\hline & CGGGAAGCGTGTACTTATCCTT \\
\hline \multirow{2}{*}{ Nanog } & TCTTCCTGGTCCCCACAGTTT \\
\hline & GCAAGAATAGTTCTCGGGATGAA \\
\hline \multirow{2}{*}{ Sacl } & GCAGCCTACGAGCATCTGAAG \\
\hline & GGACACTCGGTCAATGATGAGTA \\
\hline \multirow{2}{*}{$M D R l$} & CTGTTGGCGTATTTGGGATGT \\
\hline & CAGCATCAAGAGGGGAAGTAATG \\
\hline \multirow{2}{*}{$m A C T B$} & GGCTGTATTCCCCTCCATCG \\
\hline & CCAGTTGGTAACAATGCCATGT \\
\hline \multirow{2}{*}{$I L-10$} & GACTTTAAGGGTTACCTGGGTTG \\
\hline & TCACATGCGCCTTGATGTCTG \\
\hline \multirow{2}{*}{ Arg-1 } & TGGACAGACTAGGAATTGGCA \\
\hline & CCAGTCCGTCAACATCAAAACT \\
\hline \multirow{2}{*}{$T G F-\beta$} & CAATTCCTGGCGATACCTCAG \\
\hline & GCACAACTCCGGTGACATCAA \\
\hline \multirow{2}{*}{$i N O S$} & AGGGACAAGCCTACCCCTC \\
\hline & CTCATCTCCCGTCAGTTGGT \\
\hline \multirow{2}{*}{$I L-12$} & СCTTGCACTTCTGAAGAGATTGA \\
\hline & ACAGGGCCATCATAAAAGAGGT \\
\hline \multirow{2}{*}{$T N F-a$} & GAGGCCAAGCCCTGGTATG \\
\hline & CGGGCCGATTGATCTCAGC \\
\hline \multirow{2}{*}{$I L-6$} & CCTGAACCTTCCAAAGATGGC \\
\hline & TTCACCAGGCAAGTCTCCTCA \\
\hline \multirow{2}{*}{ hACTB } & CATGTACGTTGCTATCCAGGC \\
\hline & CTCCTTAATGTCACGCACGAT \\
\hline
\end{tabular}

